\title{
INTELIGENCIA EMOCIONAL Y SOCIAL COMO FACTOR DE DESARROLLO INTEGRAL DEL TRABAJADOR EN GAMARRA
}

Pedro L. Tito Huamaní*

pedrotito18@gmail.com

\begin{abstract}
RESUMEN
Las tensiones de la vida moderna, la competencia en el terreno individual y empresarial, la presión del reloj, la exigencia de un constante perfeccionamiento profesional, etc. Son situaciones que tienden a alterar el estado emocional de la mayoría de las personas consideradas normales, llevándolas al borde de sus propios límites físicos y psíquicos. El resultado, a menudo, es el desequilibrio emocional.

Este desequilibrio no solo afecta la vida más íntima de una persona, sino que afecta su trabajo y su desarrollo profesional, porque las emociones desempeñan un papel importante en el ámbito laboral. De la ira al entusiasmo, de la frustración a la satisfacción, cada día nos enfrentamos a emociones propias y ajenas. La clave está en utilizar las emociones de forma inteligente, para que trabajen en beneficio propio, de modo que nos ayuden a controlar nuestra conducta y nuestros pensamientos en pos de mejores resultados, lo que se conoce como inteligencia emocional.
\end{abstract}

La mejor manera de definir la inteligencia emocional es considerar que se trata de la capacidad de aplicar la conciencia y la sensibilidad para discernir los sentimientos que subyacen en la comunicación interpersonal, y para resistir la tentación que nos mueve a reaccionar de una manera impulsiva e irreflexiva, obrando en vez de ello con receptividad, con autenticidad y con sinceridad. Esto es un problema que se da en todos los ámbitos, uno de los cuales ha tenido más relevancia en los últimos años: el ámbito laboral. Pero irealmente los empresarios y los trabajadores le dan la importancia necesaria a este aspecto?

El estudio realizado precisamente nos lleva a darnos cuenta cuán importante es la inteligencia emocional como factor de desarrollo integral del trabajador peruano, fundamentalmente en el emporio empresarial de Gamarra; lo cual nos permite tener un panorama más amplio de cómo aplican ellos, si así fuera el caso, la inteligencia emocional en sus centros de labores y cómo afecta esto su desempeño.

Palabras clave: inteligencia emocional, gestión de mypes, emporio comercial de Gamarra

\footnotetext{
* Doctor en Ciencias Administrativas, Magíster en Gestión Empresarial y Licenciado en Administración. Docente Asociado de la UNMSM, Docente de Postgrado de la FCA.
} 


\section{ABSTRACT}

The stresses of modern life, competition at the individual and business, the pressure of the clock, a constant demand for professional development, etc., Are situations that tend to alter the emotional state of most normal people, taking them to the edge of their own physical and mental limits. The result is often emotional imbalance.

This imbalance not only affects the intimate life of a person, it affects their work and professional development, because emotions play an important role in the workplace. From anger to excitement, from frustration to satisfaction, every day we face-our own and others emotions. The key is to use emotions intelligently, to work for themselves, so help us control our behavior and our thoughts towards better results, which is known as emotional intelligence.

The best way to define emotional intelligence is considered that this is the ability to apply awareness and sensitivity to discern the feelings underlying interpersonal communication, and to resist the temptation that leads us to react in an impulsive, thoughtless, instead working with responsiveness, with authenticity and sincerity. This is a problem that occurs in all areas, one of which has been more relevant in recent years is the workplace. But really the employers and workers give the necessary importance to this aspect?

The study leads precisely to realize how important emotional intelligence as a development of the worker Peru, mainly in the business empire of Gamarra MSE, which allows us to have a broader picture of how they apply them, if so the case, emotional intelligence in their places of work and how it affects their performance

Keywords: Emotional Intelligence, Management Mypes, commercial Emporio Gamarra

\section{INTRODUCCIÓN}

La gran mayoría de los directivos, gerentes y supervisores tienen conciencia de la importancia de la competencia social y, en el futuro, estará entre las competencias gerenciales más importantes entre los ejecutivos, estos tendrán que tener la capacidad de transmitir a sus colaboradores una sensación de proximidad y de calor humano.

Por ello, cuando los empleados logran identificarse con la empresa y participan, siendo escuchados para tomar decisiones, adoptan los objetivos de la organización como propios y ponen todo su empeño en alcanzarlos ya que se sienten bien motivados.

Por el contrario, las emociones negativas los frenan, cuando existen disgustos por culpa del jefe, traerá como consecuencia la desmotivación y la falta de compromiso de los colaboradores ante el trabajo que realizan. Además, cuando los trabajadores temen constantemente ante la posibilidad de perder su puesto de trabajo, su capacidad para rendir en forma efectiva disminuye y se resiente.

Así el siguiente artículo trata sobre la importancia de la inteligencia emocional y social como factor de desarrollo integral del trabajador, tomando como ejemplo a las Mypes del emporio comer- cial de Gamarra, el cual es considerado como el centro comercial e industrial de ropa más grande de Sudamérica, con casi 20000 empresas en un solo lugar. Los temas a tratar son la inteligencia emocional, la cual es la capacidad de reconocer nuestros propios sentimientos, los sentimientos de los demás; además la inteligencia social que constituye una de las herramientas importantes en el mundo profesional y gerencial; también el desarrollo integral, la cual es entendida como el proceso de perfeccionamiento del hombre que implica totalidad e integralidad. Todos estos temas se detallarán en cada capítulo del marco teórico a tratar.

\section{FICHA TÉCNICA}

Este trabajo de investigación se define como descriptivo, ya que consiste en determinar la existencia de la inteligencia emocional y social en los trabajadores del emporio de Gamarra, y de ser así establecer en qué nivel se encuentra. Asimismo, se estimará la influencia de la inteligencia emocional en el puesto de trabajo de cada persona que sea objeto de estudio, a través de los niveles de autorrealización, desarrollo personal y desempeño laboral, que se determinará a través de las encuestas. 
La población total de la investigación son los trabajadores de las micro y pequeñas empresas (mypes) que conforman el emporio comercial de Gamarra, entre tiendas comerciales, fabricantes y contratistas, servicios de subcontratación y proveedores de la industria textil ${ }^{1}$. Según estadísticas del municipio de La Victoria, en Gamarra existen 17,000 empresas ${ }^{2}$. La muestra hallada de forma probabilista arrojó como resultado de 242 empresas, sin embargo, debido a limitaciones de recursos y tiempo, se tomó solo 60 de ellas para nuestro estudio.

El trabajo de campo fue llevado a cabo a través de encuestas y test dirigido a los colaboradores y jefes de las mypes seleccionadas, los cuales fueron complementados con entrevistas a los jefes y la misma observación de los miembros del equipo investigador.

\section{MARCO TEÓRICO}

"Cualquiera puede ponerse furioso, eso es fácil. Pero, ponerse furioso con la persona correcta, en la intensidad correcta, en el momento correcto, por el motivo correcto, y de la forma correcta... Eso no es fácil" - Aristóteles.

\section{INTELIGENCIA EMOCIONAL}

Según Goleman, la inteligencia emocional es “... la capacidad de reconocer nuestros propios sentimientos, los sentimientos de los demás, motivarnos y manejar adecuadamente las relaciones que sostenemos con los demás y con nosotros mismos. Se trata de un término que engloba habilidades muy distintas -aunque complementarias- a la inteligencia académica, la capacidad exclusivamente cognitiva medida por el cociente intelectual..."

Para Coopers y Sawaf, "La inteligencia emocional es la capacidad de sentir, entender y aplicar eficazmente el poder y la agudeza de las emociones como fuente de energía humana, información, conexión e influencia".

Si observamos las definiciones anteriores, todo se centra en las emociones, es decir, todo gira de cómo se identifica, se controla y como se puede utilizarlas de la forma más productiva. Tanto con nuestro interior así como nos comportamos con los demás. Por eso, las emociones juegan un papel importante en nuestras vidas pues generan estímulos y energías para poder alcanzar los objetivos que nos trazamos. Pero también puede generarnos frustraciones que inhiban nuestros pensamientos y acciones. Con respecto a los demás nos ayuda a transmitir entusiasmo y reclutar seguidores de nuestros proyectos; aunque, también pueden ocasionarnos conflictos y rechazos, si no sabemos controlarlas y manejarlas de manera adecuada, es decir, en forma inteligente.

Dentro de la inteligencia emocional encontramos tres aspectos: Aspectos fisiológicos de las emociones son aquellas reacciones que se presenta en el organismo del ser humano como: las alteraciones en la circulación, los cambios respiratorios y las secreciones glandulares; aspecto social son reacciones que se presentan durante el contacto con nuestro entorno social como cólera: esta se presenta cuando tenemos una frustración al no obtener lo que deseamos o necesitamos, temor, se presenta cuando reaccionamos de manera inesperada ante una situación que llega de manera rápida; y las emociones agradables son aquellas emociones sociales que tienen el carácter de ser agradables y liberadoras de tensión y excitación en las personas, como, gozo, júbilo, amor y risa, etc.

\section{INTELIGENCIA SOCIAL}

La noción de "inteligencia social" fue propuesta por primera vez por el psicólogo Howard Gardner, que constituye una de las herramientas importantes en el mundo profesional y gerencial. Por eso, todo líder debería agudizar al máximo su inteligencia social con el fin de perfeccionar su capacidad de entender patrones de interacción social, su ingenio político y sus relaciones.

Durante nuestra vida diaria podemos observar que estamos en constante contacto con nuestros padres, amigos, pareja, etc. y esto es lo que nos caracteriza como ser sociable. A continuación algunas preguntas que responde la inteligencia social.

\footnotetext{
1 Portal Gamarra. “¿Qué es Gamarra?”. 26 de abril del 2010. En: http://www.gamarra.com.pe/gamarra/

2 Diario Gestión. "Masivo ingreso de ropa china subvaluada a Gamarra”. 25 de octubre del 2009. EN: http://gestion.pe/noticia/359787/masivoingreso-ropa-china-subvaluada-gamarra.

3 http://www.degerencia.com/articulo/inteligencia_emocional_para_el_trabajo_gerencial

4 Ibíd.
} 
1. ¿Qué es la inteligencia social y cómo podemos desarrollarla?

2. ¿Cómo manejar los sentimientos cuando uno está en relación con otras personas?

3. ¿Por qué las relaciones humanas satisfactorias se traducen en un mejor estado de salud?

4. ¿Cómo podemos lograr que grupos divididos por el prejuicio y el odio vivan juntos en paz?

5. ¿Cuál es el lado oscuro de la inteligencia social?

En conclusión, la inteligencia social se puede definir como una capacidad humana que tiene para relacionarse con su entorno.

\section{DESARROLLO INTEGRAL}

\section{Conceptos sobre el desarrollo}

Cuando hablamos de desarrollo lo relaciona mos más con disfrutar de bienes materiales. Es decir, bienes que satisfacen necesidades superfluas. Por eso el "tener más" aparece como el objetivo importante del desarrollo. Existe una teoría que es más aceptada en Latinoamérica que habla del subdesarrollo en relación directa con la opresión y la dependencia. Por esta razón existe una necesidad de vencer la injusta situación con respecto a la liberación económica, social y política.

\section{El desarrollo integral: proceso de perfeccionamiento humano}

El proceso de perfeccionamiento del hombre implica totalidad, integralidad. El perfeccionamiento del hombre consiste concretamente en la realización de este en todas sus dimensiones, y no solo del hombre considerado como individualidad, sino de todos los hombres, y del mundo como campo en el cual se ejerce la acción humana.

El desarrollo integral se caracteriza por ser un proceso. Este proceso avanza hacia una dirección ideal. Por lo que se supone que hay una serie de mejoramientos progresivos. Es decir, tener un paso adelante para llegar a la perfección de los hombres y de la sociedad. Como todo proceso implica el avanzar y no imprescindiblemente el llegar.

El desarrollo integral del ser humano a nivel mundial según el Programa de de las Naciones Unidas para el desarrollo. Nuestro país se encuentra ubicado en el puesto 63, es decir, dentro de los 4 niveles que son: desarrollo humano muy alto que son 42 países ocupando como el primer lugar Noruega; desarrollo humano alto, va desde el puesto 43 hasta el 85, Desarrollo humano medio del 86 hasta el 127 y el desarrollo humano bajo. (127-169) ocupando el último lugar Zimbabwe.

"El Índice de Desarrollo Humano (IDH) es una medida sinóptica del desarrollo humano. Mide el progreso medio conseguido por un país en tres dimensiones básicas del desarrollo humano: disfrutar de una vida larga y saludable, acceso a educación y nivel de vida digno". ${ }^{5}$

\section{ANÁLISIS DE LA INFORMACIÓN}

En el desarrollo de este capítulo se consideran 60 empresas del emporio comercial de Gamarra, donde se aplicaron dos tipos de encuestas, una dirigida a los trabajadores y la otra hacia los jefes o encargados de los trabajadores encuestados.

\section{A. Encuesta a los jefes:}

En esta primera parte, se muestra el análisis de los resultados obtenidos de las encuestas realizadas a los 60 jefes o dueños de las empresas que fueron objeto del presente estudio.

Según los jefes o encargados encuestados (Gráfico $\mathrm{N} .^{\circ} 1$ ), el $41 \%$ ha presenciado dos conflictos con los colaboradores durante el último mes, y que en varias oportunidades han tenido que involucrarse para poder resolverlo, mientras que un $26 \%$ manifiesta que en el trabajo no ha habido ninguna clase de conflictos; seguidamente un $21 \%$ manifiesta que ha tenido un conflicto con algún trabajador pero que no ha sido de mucha importancia y por último un $12 \%$ manifiesta que ha tenido más de 3 conflictos en su área de trabajo.

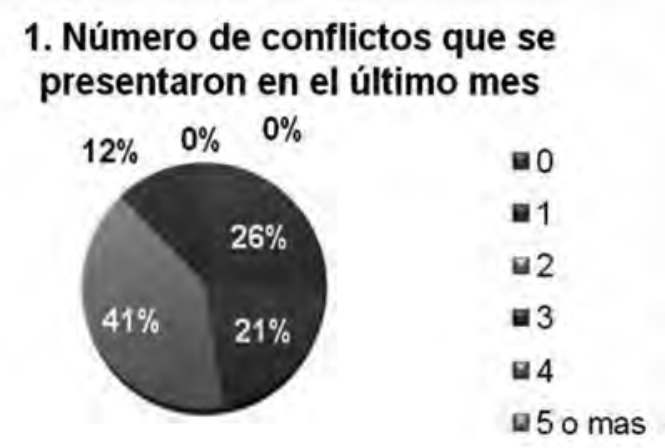

GRÁFICO Nº 01

5 http://hdr.undp.org/es/estadisticas/indices/ 
En el gráfico $\mathrm{N} .^{\circ} 2$, se muestran los resultados del número de capacitaciones recibidas por sus trabajadores en los últimos 12 meses. Al respecto, los encargados manifestaron que el $50 \%$ no ha realizado capacitaciones, el $34 \%$ solo una vez, el $3 \%$ solo 2 veces y el $13 \%$ solo 3 veces. Por ello, se concluye que por lo menos una vez al año se realizan capacitaciones para el desarrollo de los trabajadores.

\section{2. ¿Cuántas capacitaciones a sus trabajadores ha realizado los últimos 12 meses?}

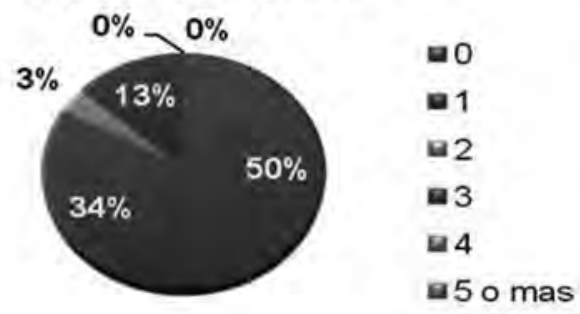

$$
\text { GRÁFICO N. }{ }^{\circ} 2
$$

En relación con el promedio de asistencias a las capacitaciones, tal como se muestra en el gráfico N. ${ }^{\circ}$, el $70 \%$ de los encargados o jefes encuestados manifiestan que la asistencia porcentual promedio de los trabajadores a las capacitaciones es de $26 \%$ al $50 \%$, mientras que el $30 \%$ manifiesta que la asistencia porcentual promedio es de $0 \%$ al $25 \%$. Lo que se puede concluir que los trabajadores no les toman la debida importancia a ellas o que cuando se presentan la oportunidad no son comunicadas.

\section{Diga Usted que porcentaje promedio de trabajadores asistieron a las capacitaciones}

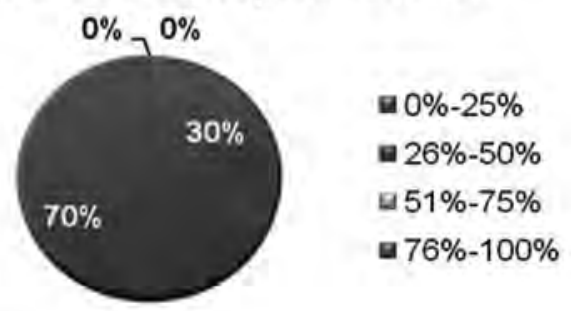

GRÁFICO N. ${ }^{\circ} 3$

En relación al movimiento de personal (Gráfico N. ${ }^{\circ} 4$ ), el $33 \%$ de los encuestados dicen que lo realizan anualmente, mientras que el $11 \%$ lo realiza semestralmente, el 9\% lo hace trimestral, el $21 \%$ no hace este movimiento, y el $26 \%$ puede que lo haga mensual, bimestral dependiendo de la política que tenga la empresa. De ello se concluye que en las empresas de Gamarra la mayoría ejecuta el movimiento del personal.

\section{Dicen los expertos que el movimiento de personal hace que los trabajadores no se especialicen en su área de trabajo o caso contrario caerian en la rutina. Usted ¿con qué frecuencia realiza movimiento a su personal?}

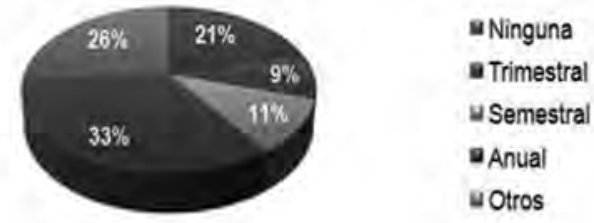

GRÁFICO N. ${ }^{\circ} 4$

El Gráfico N. ${ }^{\circ} 5$, refleja los resultados del nivel de satisfacción que muestran los jefes o administradores de sus colaboradores. Cabe resaltar que el $85 \%$ de los jefes califica la labor de los colaboradores como bueno y un $15 \%$ como muy bueno. En suma la percepción que tienen los jefes entre bueno y muy bueno equivalen al $100 \%$.

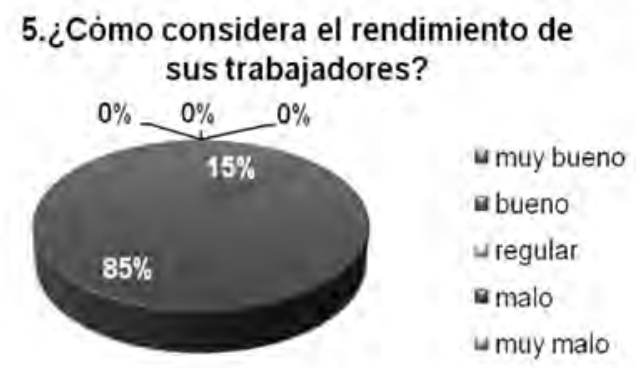

GRÁFICO N. ${ }^{\circ} 5$

De la misma forma, el Gráfico N. ${ }^{\circ} 6$ muestra que el $4 \%$ de los jefes encuestados consideran que sus trabajadores alcanzan un promedio de 0 a $25 \%$ de sus objetivos. Asimismo, el $63 \%$ afirma que alcanzan los objetivos de la empresa en una magnitud de $26-50 \%$, el $33 \%$ de ellos considera una magnitud de 51-75\% y en ningún caso consideró la opción de 76 a $100 \%$ de los objetivos.

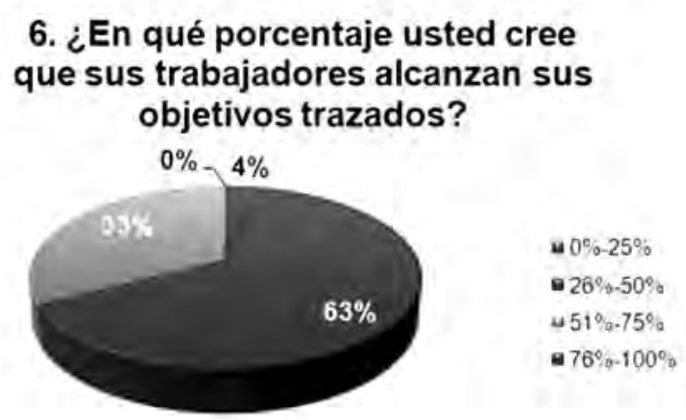

GRÁFICO N. ${ }^{\circ} 6$ 
El Gráfico N. 7 muestra que un $56 \%$ de los jefes considera que a lo largo del proceso productivo de su empresa se incurre en una merma promedio entre $0-25 \%$ y un $44 \%$ manifestó que los desperdicios son en un mayor porcentaje, aproximadamente entre $26-50 \%$. Ello implica que existe una carencia en el uso oportuno de los recursos; esta situación se puede deducir de la poca capacitación y asesoramiento de los empleados para desarrollar eficientemente sus labores, si bien el porcentaje aproximado de desperdicios no es alto, es necesario disminuir al mínimo esta merma.

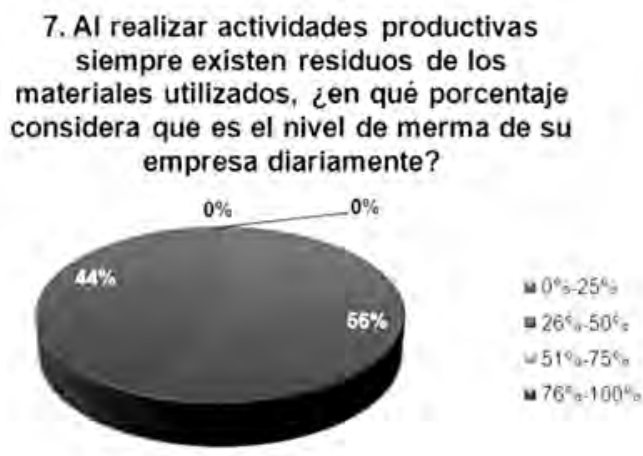

\section{GRÁFICO N ${ }^{\circ} 07$}

En el gráfico $\mathrm{N}^{\circ} 08$, el $82 \%$ de los jefes ha optado por la opción A, lo que demuestra la firmeza de ellos en querer alcanzar sus metas en colaboración con sus trabajadores mostrando que los jefes tienen un control de sus emociones. Por otro lado un $18 \%$ optó por la opción B lo cual muestra que tienen un bajo control de sus emociones; es decir, que son sensibles ante algún problema que se presente en el trabajo , no hay mucha firmeza a la hora de tomar las decisiones.

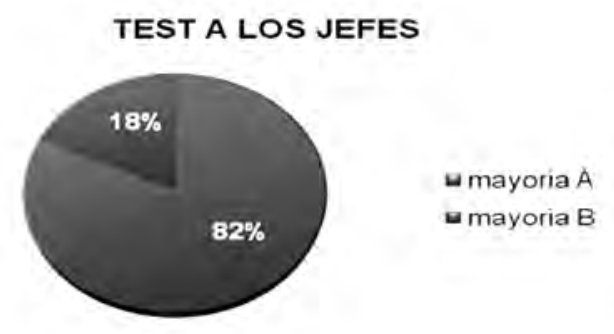

GRÁFICO N ${ }^{\circ} 08$

\section{B. Encuesta a los trabajadores:}

En este apartado se muestra el análisis de los resultados obtenidos de las encuestas realizadas a 60 colaboradores de las empresas que fueron objeto del presente estudio
El gráfico $\mathrm{N}{ }^{\circ} 9$ demuestra que el mayor porcentaje de los colaboradores encuestados no ha presentado ningún tipo de conflictos en el último mes con un 55\%; sin embargo, el $15 \%$ aseguró haber presentado al menos un conflicto; el 27\%, dos conflictos y solo un 3\% manifestaron haber presentado por lo menos 3 conflictos en su lugar de trabajo. Se puede concluir que hay un aparente estado de armonía en el lugar de trabajo, ya que más de la mitad de los encuestados no ha presenciado ni participado de ningún tipo de discusiones con algún compañero o jefe.

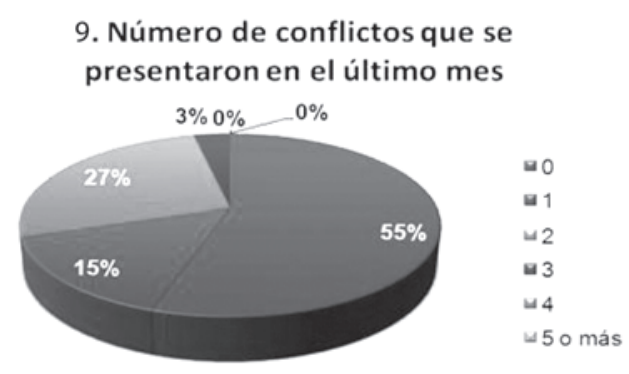

\section{GRÁFICO N. ${ }^{\circ} 9$}

Para determinar si las mypes de Gamarra ayudan o influyen en el desarrollo del trabajador, se les consultó sobre el número de capacitaciones recibidas a los largo del tiempo que labora en sus empresas (Gráfico $\mathrm{N}^{\circ} 10$ ), dando como mayor resultado con un $27 \%$ con 2 capacitaciones, seguido por un $22 \%$ que recibieron solo 4 , en el caso de los colaboradores que recibieron 3 y 6 capacitaciones ambos alcanzaron el $17 \%$ del total y solo $1 \%$ recibió 5 capacitaciones. En este sentido, existe la preocupación por dichas empresas para que sus empleados sean calificados y competitivos dentro de la organización a pesar de que en algunos casos sea en mayor proporción que en otros.

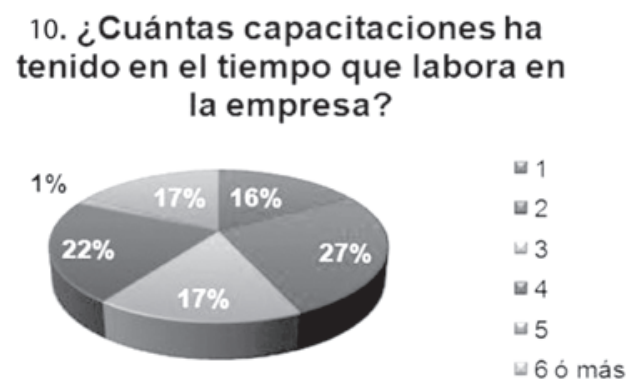

\section{GRÁFICO N ${ }^{\circ} 10$}

En el gráfico $\mathrm{N}^{\circ} 11$, se aprecia que el mayor porcentaje de las empresas ha realizado por lo menos una capacitación a los largo de los últimos 12 
meses, el $10 \%$ solo 1 , el $23 \%$ aproximadamente 2 , en un 5\% para los casos en que la empresa realizó entre 3 y 4 capacitaciones. Como contraparte hay un $57 \%$ que manifiestan no haber recibido ninguna capacitación. Tales resultados se explican ya sea por una falta de preocupación por parte de los jefes o por la poca facilidad que el Estado viene brindando a este sector para realizar este tipo de actividades.

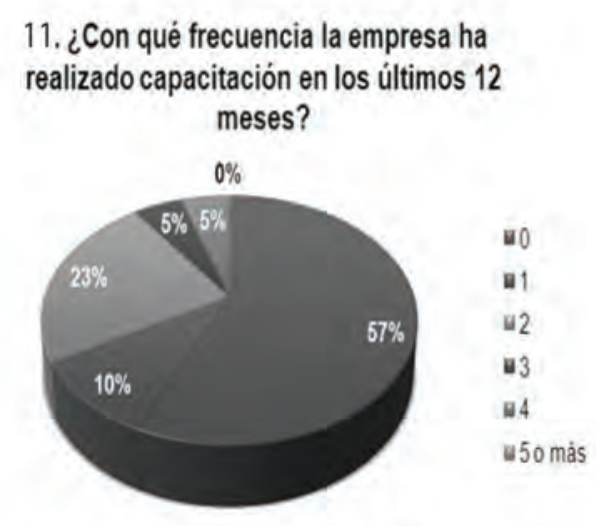

GRÁFICO N ${ }^{\circ} 11$

El gráfico $\mathrm{N} .^{\circ} 12$ muestra el nivel de asistencias de los trabajadores a las capacitaciones en los últimos 12 meses. El 70\% del total de los colaboradores encuestados manifestó haber asistido a por lo menos una de las capacitaciones brindadas por la empresa, lo que permite apreciar que ha habido un número determinado de personas que no han asistido a todos los adiestramientos que la organización ofreció; por ende, el porcentaje de asistencia disminuyó en las demás circunstancias, siendo el $8 \%$ los asistentes a 2 capacitaciones, $18 \%$ a 3 , y en los casos de 4 y 5 adiestramientos se registró solo el $2 \%$ de asistencia en ambos casos.

12. ¿Cuántas de las capacitaciones realizadas los últimos 12 meses asistió?

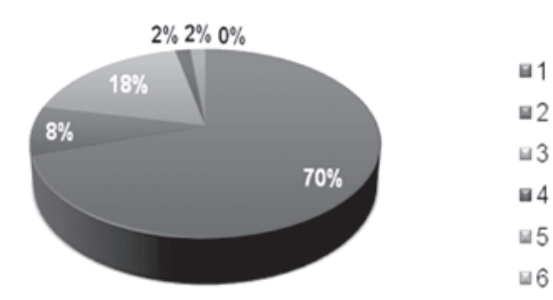

GRÁFICO N ${ }^{\circ} 12$

El gráfico $\mathrm{N} .^{\circ} 13$ refleja el nivel de formación académica de los asistentes a las capacitaciones. Así, se observa que los colaboradores con secundaria completa asistieron en un $42 \%$, en tanto que con el nivel primario asistieron en un 25\%. Un $23 \%$ de colaboradores han concluido o están cursando estudios de nivel técnico y el 10\% de los asistentes tienen el nivel de formación superior universitario.

\section{3. ¿Cuál es su grado académico?}

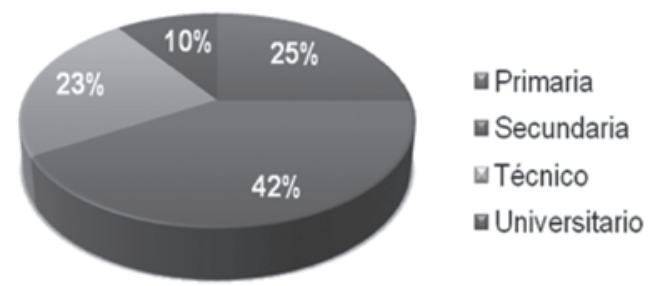

\section{GRÁFICO N ${ }^{\circ} 13$}

El panorama es alentador, pues, a pesar de que sea en menor proporción, existe un número de colaboradores que aspira a incrementar su nivel académico al cursar o haber concluido estudios superiores, ya que en un futuro próximo estas personas pueden crear nuevos negocios con la experiencia que están adquiriendo en la empresa.

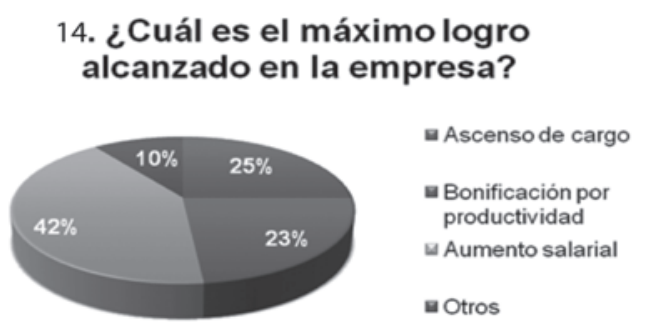

\section{GRÁFICO N ${ }^{\circ} 14$}

En lo que respecta al logro alcanzado por los trabajadores en sus respectivos centros de labores (Gráfico $\mathrm{N}^{\circ} 14$ ), el $42 \%$ de los trabajadores respondió que el máximo logro se dio mediante un aumento salarial, el $23 \%$ respondió que se dio mediante una bonificación por la productividad, esto se da especialmente en épocas de campaña en Gamarra cuando se realizan una gran cantidad de pedidos y la mayoría de empresas empieza a producir en grandes cantidades; por el contrario, el 25\% refirió como máximo logro un ascenso en el cargo, esto va de acuerdo con el desempeño que se ha tenido en la empresa.

Respecto a la satisfacción con el trabajo que realiza (Gráfico N. ${ }^{\circ} 15$ ) la mayoría de los trabajadores (78\%) manifestó que se encuentra satisfecho, ya sea por el buen trato de su jefe, por las adecuadas 
condiciones en las que realiza su labor, o la buena remuneración que percibe; sin embargo, el 22\% manifestó no estar satisfecho con el trabajo que realiza ya sea porque es un trabajo muy cansado, de constante estrés o porque las condiciones no son las adecuadas.

\section{Se siente satisfecho con el trabajo que realiza?}

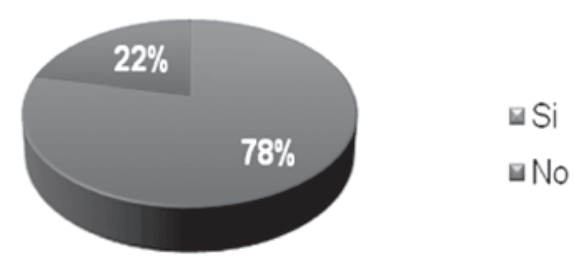

\section{GRÁFICO N. ${ }^{\circ} 15$}

En relación al Test de Inteligencia Emocional aplicado a los trabajadores (Gráfico $\mathrm{N}^{\circ} 16$ ), el 52\% de ellos han elegido la opción A para la situación planteada, esto refleja que hay un buen nivel de inteligencia emocional en los trabajadores de Gamarra, lo cual indica que ellos tiene la capacidad para resolver problemas, manejan su estrés y los conflictos que se puedan presentar, de tal manera que no afecten su desempeño en la empresa, sin embargo, es preocupante que hay un $48 \%$ de los trabajadores que tuvo mayoría de preguntas B lo cual indica que sus decisiones no son muy asertivas, no saben manejar sus emociones y eso puede influir mucho en el trabajo y en su relación con sus compañeros.

\section{TEST A LOS TRABAJADORES}

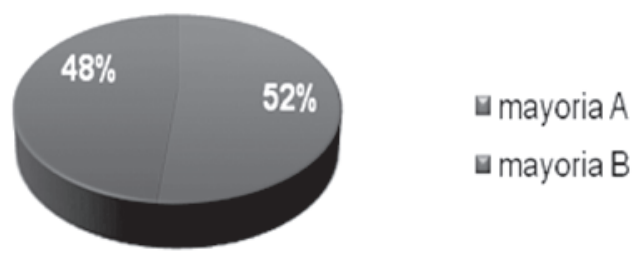

GRÁFICO N. ${ }^{\circ} 16$

\section{CONCLUSIONES}

1. La inteligencia emocional es un aspecto que ha venido cobrando cada vez más relevancia a medida que se ha dado más importancia al factor humano dentro de la organización, esto debido a que muchos jefes ya son conscientes de que el desempeño de sus trabajadores es influenciado por sus emociones y la manera en cómo se sienten, sobre todo en trabajos que están expuestos a constante estrés, se hace necesario que tanto trabajadores como jefes puedan ser inteligentes emocionalmente y lidiar con todas las tensiones o conflictos que se pueda vivir en el trabajo, ya que es esa la verdadera clave del éxito en una empresa más allá de los ingresos que se perciben pues el elemento humano es el elemento más importante.

2. La capacidad de rendimiento en equipo establece una estrecha relación entre el rendimiento y la cohesión del mismo. La competencia profesional, la creatividad, los conocimientos técnicos de cada uno de los componentes solo pueden desplegarse por completo cuando el equipo trabaja en un clima armónico. El aporte de cada miembro del grupo al éxito del equipo depende, de forma decisiva, de sus cualidades emocionales.

3. De los datos aportados por esta investigación podemos afirmar que dependiendo del estado de ánimo de los trabajadores puede aumentar o disminuir la productividad. Cuando los directivos de la organización desarrollan su competencia social promueven bienestar y contacto humano con el resto del personal que integra la organización.

4. El grado de desarrollo en los trabajadores encuestados que laboran en el emporio comercial de Gamarra es medio; si bien existe el apoyo de las mypes por brindar capacitaciones a su personal, éstas son mínimas y poco frecuentes, lo cual genera que mucho de los puestos de trabajo sean rutinarios y exhaustivos ya que no cuentan con nuevas técnicas y conocimientos que aceleren el proceso de producción.

5. Por otro lado, se apreció a lo largo de la investigación que muchos de los colaboradores están cursando o han concluido estudios técnicos y en otros casos universitarios; esto demuestra que existe un grado de esfuerzo y emprendimiento en cada uno de ellos por forjarse un futuro más próspero al alcanzar nuevos grados académicos. Por ende, se puede deducir que muchos de estos trabajadores esperan crecer en dicho tipo de negocio, ya sea en la misma empresa o empezando una nueva, lo cual será muy beneficioso para la autorrealización personal de cada uno de estos trabajadores emprendedores. 


\section{RECOMENDACIONES}

1. Las mypes de Gamarra deben tomar conciencia de la importancia que tienen sus trabajadores en la empresa e incentivarlos a crecer y no quedarse en un solo puesto por años. Es recomendable que los microempresarios brinden capacitaciones gratuitas a sus colaboradores con mayor frecuencia y a un mayor número de personas, de tal manera que esto ayude a los empleados a obtener nuevos conocimientos y volviéndolos más expertos en su área de trabajo; por otro lado, esto beneficiará a la empresa al incrementarse la productividad y la calidad del producto que elaboran.

2. Asimismo, los jefes deben fomentar un mayor involucramiento e identificación del personal hacia la empresa, pues de nada servirá que se les facilite asesoramiento si los trabajadores carecen de interés y no asisten a estas actividades; por ello, es importante la motivación constante ya sea por logros, competencia, afiliación o autorrealización, según convenga a cada organización y permita incrementar los niveles de inteligencia emocional en cada individuo.

3. La inteligencia emocional no es ahogar las emociones, sino dirigirlas y equilibrarlas. Ejercer un autodominio emocional no significa negar o reprimir los verdaderos sentimientos. Los estados de ánimo "malos", por ejemplo; tienen su utilidad: el enojo, la tristeza y el miedo pueden ser una intensa fuente de motivación, sobre todo cuando surge del afán de corregir una situación de adversidad, una injusticia o inequidad. La tristeza compartida puede unir a la gente. Y la urgencia nacida de la ansiedad (mientras no sea sobrecogedora) puede acicatear el espíritu creativo. Por ello, es importante que dentro de las organizaciones, los jefes o encargados que tienen a su mando un equipo de trabajo y se presente por varias circunstancias, se recomienda que antes de actuar deben pensar con inteligencia emocional y hará que las reacciones sean más inteligentemente llevadas.

4. Teniendo en cuenta que el cociente intelectual determina lo que sabe un ejecutivo, la inteligencia emocional determina lo que hará; es decir, el cociente intelectual es lo que permite entrar en una organización, pero la inteligencia emocional es lo que permite crecer en esa or- ganización y convertirse en líder, entonces es de suma importancia su apropiado desarrollo en cada uno de los trabajadores tanto para él mismo como para la empresa en que labora.

4. Para poder mejorar situaciones que ocurren en la empresa debido a una falta de inteligencia emocional como reacciones violentas, conflictos, desgano, apatía, es necesario aprender a detectar ganancias personales en todo lo que se emprende. Esto no implica volverse un interesado. Simplemente se tiene que aprender a buscar motivos personales que generen el entusiasmo que se necesita para realizar las tareas. Si la meta o el resultado final a lograr se ve muy lejano o muy difícil de conseguir, se debe poner metas alcanzables en el corto plazo cuya suma lleven al resultado final que se desea. Un ejemplo de ello es pensar que al realizar una actividad de bordado en una empresa textil se está adquiriendo todos los conocimientos necesarios para mejorar el desempeño propio y quizás en un futuro abrir una empresa propia.

5. Es necesario que en el ambiente laboral exista más confianza entre jefes y colaboradores, ya que esto permitirá la buena comunicación entre ellos y así se podrá realizar el trabajo de la manera correcta y de esta manera puedan lograr los objetivos trazados. Además es necesario que haya mucha más motivación hacia los colaboradores de la empresa ya que esto permitirá una mayor identificación por parte de ellos con la empresa.

\section{LITERATURA CITADA}

BAR-ON, R. y Otros. (2000). Emotional expression and implications for occupational stress: An application of Emotional Quotient Inventory (EQ-i). Personality and Individual Differences. Holanda.

CLORE, G. L. y ORTONY, A. (2000). Cognition in emotion: Always, sometimes, always or never. Nueva York. Oxford University Press.

COOPER, R. K. y SAWAF, A. (1997). Executive EQ: emotional intelligence in leadership and organizations. Nueva York: Gosset, Putnam.

GOLEMAN, Daniel. (1995). Emotional intelligence: Why it can matter more than IQ. New York: Bantam Books. 
HERNÁNDEZ SAMPIERI, Roberto y otros. (1991) . Metodología de la Investigación. McGraw Hill Interamericana. México, D.F.

KERLINGER, FN. (1979). Enfoque conceptual de la investigación del comportamiento. Nueva Editorial Interamericana. México, D.F.

SALOVEY, P. y MAYER, J. D. (1990). Emotional intelligence. Imagination, cognition and personality. New York.

\section{B. INTERNET}

http://metodoinvestigacion.files.wordpress. com/2008/05/como_escribir_tesis_ana-h.pdf

http://www.inteligencia- mocional.org/trabajoyempresa/lainteligenciaemocionalyeltrabajoenequipo.htm http://www.inteligencia-emocional.org/preguntas_frecuentes/index.htm

http://www.cybertesis.edu.pe/sisbib/2008/arredondo_bd/pdf/arredondo_bd.pdf

http:/hdr.undp.org/es/estadisticas/indices/

http://www.banrepcultural.org/blaavirtual/ciencias/sena/administracion/realizacion/irealiz7a.htm

http://168posibilidadesdelalma.blogspot. com/2010/07/la-inteligencia-social.html

http://es.wikipedia.org/wiki/Conciencia_ (psicolog\% $\%$ C3\%ADa) 